\title{
Carbon nanotube enabled superhydrophobic surfaces
}

\author{
Yugam Singla, Karan Prihar and Prabhat K Agnihotri* \\ Mechanics of Advanced Materials laboratory (MAdMatLab), School of Mechanical, Materials and \\ Energy Engineering, Indian Institute of Technology Ropar, Rupnagar-140001, Punjab, India \\ *Email: prabhat@iitrpr.ac.in
}

Due to the broad range of potential applications, superhydrophobic surfaces (surfaces with water contact angle (CA) higher than $150^{\square}$ ) have recently attracted much interest. Superhydrophobic surfaces exhibit extremely high water repelling behavior due to large values of CA and a low sliding angle (less than $\left.10^{\circ}\right)$. Water drops bounce on these surfaces if dropped from a height. Lotus leaves are the most exciting superhydrophobic surfaces found in nature. The morphology of lotus leaves shows that hierarchical nanostructuring on its surface is primarily responsible for their super hydrophobic behavior. Hence, low surface free energy and high surface roughness are the two main requirements to fabricate super hydrophobic surfaces.

Inspired by this observation, efforts are made in the present work to create an artificial lotus like surface using patterned growth of carbon nanotubes (CNTs) using vacuum chemical vapor deposition technique. $\mathrm{Ni}$ catalyst is deposited using thermal evaporation method. CNTs are preferred due to their very high surface area, extremely high stiffness and nanoscale diameter. Plane silicon wafers are used as substrate to grow hierarchical nanostructure of CNTs. It has been shown that CA values of as high as $172^{\circ}$ can be obtained using proposed methodology. Next, a parametric study is performed to characterize the effect of CNT pillars height and spacing (pattern type) on the hydrophobicity of the nanostructured silicon wafer surface. It is observed that the contact angle can be tuned through patterned growth of CNTs on plane silicon wafers, as shown in Figure 1.

The sliding angle of $\sim 9^{\circ}$ is obtained for the developed surfaces indicating a Cassie-Baxter configuration of drops on nanostructured silicon wafers. This is one of the important aspect as far as the application of superhydrophic surfaces in self cleaning surfaces is concerned.

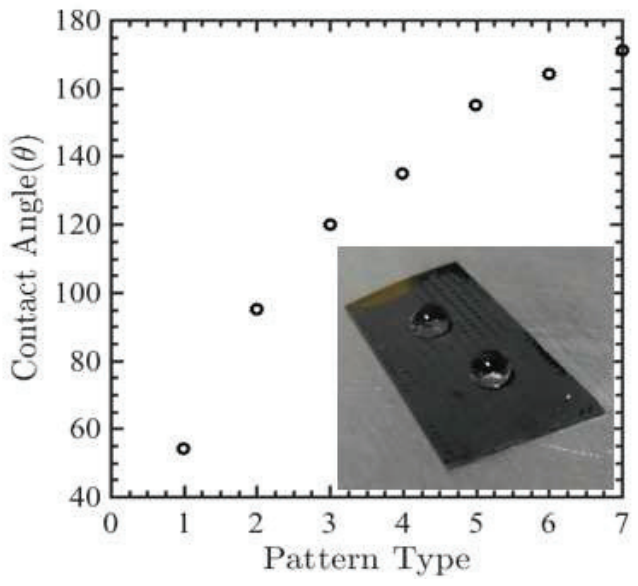

Figure 1: Variation of contact angle as a function of CNT pattern type. Inset shows water drops resting on nanostructured silicon wafer without wetting it

The viscoelastic behavior of patterned CNT pillars is also investigate by carrying out nanoDMA in Hysitron Triboindenter in the frequency range of 2-200 Hz. It is shown that the dynamic behavior of CNT pillars is a function of CNT pillar height and spacing. This study opens an opportunity to use these CNT nanostructures to provide damping in MEMS and other miniaturized electronic devices. The future directions regarding processing, characterization and application of superhydrophobic and hierarchical nanostructured surfaces is also discussed.

\section{Reference}

1. Bharat Bhushan, Michael Nosonovsky, "Green Tribology-Bio mimetics, Energy Conservation and Sustainability" The Royal Society 368 (2010) 46774694. 